Asian Spine Journal

Vol. 2, No. 1, pp 15 21, 2008

\title{
Characterization of Magnetic Resonance Images for Spinal Cord Tumors
}

\author{
Jae Yoon Chung, Jae Joon Lee, Hyun Jong Kim, Hyung Yeon Seo \\ Department of Orthopedic Surgery, Chonnam National University Hospital, Gwangju, Korea
}

\begin{abstract}
Study Design: Retrospective study MR images for spinal cord tumors.
Purpose: To analyze the characteristics of MR images for spinal cord tumors, which were then verified at surgery or biopsy. Overview of Literature: MR images are often used as the primary diagnostic imaging tool and the preoperative study of choice. The need for biopsy may be obviated because of increasingly accurate preoperative histologic diagnosis by MR images.
\end{abstract}

Methods: The study group consisted of 39 patients who had undergone MR imaging for preoperative evaluation of spinal cord tumors between September 1989 and February 2008. All patients had operations for spinal cord tumors, which were confirmed at biopsy. Of the 39 patients, 18 were men, and 21 were women. The average follow-up period was 23.8 months. The mean patient age was 46.6 years.

Results: Diagnoses included neurilemmoma (19 cases), neurofibroma (4 cases), meningioma ( 5 cases), hemangioma ( 3 cases), giant cell tumor ( 1 case), ganglioneuroma ( 1 case), lymphoma (1 case), neuroblastoma (1 case), and metastatic tumor from the prostate $(1$ case). The remaining 3 cases were composed of arachnoid cysts ( 2 cases) and a vascular malformation (arteriovenous malformation, 1 case).

Conclusions: MR images are the preoperative modality of choice in the evaluation of spinal cord tumors. MR images can narrow the differential diagnosis and guide surgical resection.

Key Words: Magnetic resonance imaging, Spinal cord, Tumor

\section{Introduction}

Spinal cord tumors are wide-ranging in their histologic appearance and have an even wider range of clinical symptoms and prognostic features. They are relatively rare, but if left untreated, can cause serious neurological deficits and disability. An accurate diagnosis is therefore crucial in determining prognosis and directing therapy. Significant advances have occurred in radiological imaging of spinal cord tumors over the past 20 years. Imaging is now a crucial component of diagnosis and treatment planning. A variety of imaging modalities are widely available. Computed tomography (CT) and magnetic resonance (MR) are the most helpful imaging tools for the early detection of spinal cord tumors. MR images are often used as the primary diagnostic imaging tool and are the preoperative study of choice. The need for biopsy may be obviated because of the increasingly accurate preoperative histologic diagnosis, as obtained through MR images. Brotchi et al. ${ }^{1}$ reported that MR images and histologic diagnoses are concordant $70 \%$ of time. We studied the characteristics of MR images in patients with spinal cord tumors, which were then verified at surgery or biopsy.

\section{Materials and Methods}

The study group consisted of 39 patients who underwent MR imaging for preoperative evaluation of spinal cord

Corresponding author: Jae Joon Lee, MD

Department of Orthopedic Surgery, Chonnam National University Hospital

8 Hakdong, Gwangju, 501-757, Korea

Tel: +82-62-227-1640, Fax: +82-62-225-7794, E-mail: sanggunlee@lycos.co.kr 
tumors between September 1989 and February 2008. All patients also had operations (laminoplastic tumor excision) for spinal cord tumors, which were confirmed by biopsy. Of the 39 patients, 18 were men, and 21 were women. The average follow-up period was 23.8 months. The mean patient age was 46.6 years. All MR images were obtained on a 1.0-T (GE medicine) or 1.5-T (GE medicine) unit with a spine surface coil. T1-weighted (repetition time msec/echotime msec, 400 600/8 22) and T2-weighted $(2,000 \sim 3,500 / 20 \sim 104)$ spine echo images were obtained in the sagittal plane, with $5 \mathrm{~mm}$ section thickness. Axial images were obtained in any area of the spine where sagittal images demonstrated abnormal findings. Contrast-enhanced images were obtained in all patients after injection of 0.1 $\mathrm{mmol} / \mathrm{kg}$ of gadolinium diethylene triamine acid ( $\mathrm{Gd}$ DTPA) dimeglumine solution. Contrast-enhanced images were obtained using the same T1-weighted pulse sequences as in the non-enhanced studies. Lesion signal intensity was compared to adjacent normal tissue (i.e. bone, soft tissue). Other evaluated features included homogenicity and heterogenicity in T1-,T2-, and Gd-DTPA-enhanced images. All patients had a pathologic diagnosis and were grossly evaluated for focal hemorrhage, cystic change, and myxoid change.

\section{Results}

Spinal cord tumors were classified as intramedullary, intradural/extramedullary, and extradural on the basis of their location and histology. Three cases were extradural in location, and 36 cases were intradural/extramedullary in location. There were no intramedullary lesions.

Table 1 summarizes the patient data and the MR images. Spine cord tumors were identified in 36 of 39 cases. Diagnoses included neurilemmoma (19 cases), neurofibroma (4 cases), meningioma (5 cases), hemangioma (3 cases), giant cell tumor of the tendon sheath (1 case), ganglioneuroma (1 case), lymphoma (1 case), neuroblastoma (1 case), and metastatic tumor from the prostate (1 case). The remaining 3 cases were composed of arachnoid cysts ( 2 cases) and a vascular malformation (arteriovenous malformation [AVM], 1 case). All diagnoses were established histologically (biopsy and definitive surgery). Thirty-five tumors showed contrast enhancement with Gd-DTPA. In two cases (neurilemmoma, AVM), contrast-enhanced images were not obtained at the local hospital. Neither arachnoid cyst patient received intravenous Gd-DTPA for enhanced imaging. Heterogenic enhancement was detected on Gd-DTPA images in 9 cases, and rim enhancement was detected in 3 cases. All cases with rim enhancement had cystic changes, and 9 cases with heterogenic enhancement had focal hemorrhage, focal necrosis, and myxoid change on pathologic macroscopic examination. One case had combined cystic change and focal hemorrhage.

\section{Discussion}

\section{Nerve sheath tumors}

Tumors of the spinal nerve sheath, neurofibroma, neurilemmoma, and schwannoma represent $30 \%$ of intradural/ extramedullary tumors ${ }^{3}$. Neurilemmomas and neurofibromas are histologically distinct tumors, but are indistinguishable by imaging. Both are composed of Schwann cells. Neurilemmomas almost always arise from the dorsal sensory roots and form well-encapsulated firm masses that compress adjacent tissue, without invading the involved nerve. Neurofibromas are more complex and are composed of Schwann cells mixed with fibroblasts; they involve the parent nerve. They are unencapsulated, often fusiform, and enlarge within the nerve itself.

Intradural neurilemmomas tends to have signal intensity equal to, or less than, that of the spinal cord on T1-weighted images and mild to marked hyperintensity on T2-weighted images. Focal areas of even greater hyperintensity on T2weighted images often correspond to cystic portions, whereas hypointensity may represent hemorrhage, dense cellularity, or collagen deposition. Tumor heterogenicity does not necessarily indicate malignant change ${ }^{4,5}$. In our cases, neuroblastomas demonstrated isointensity or intermediate signal intensity on T1-weighted images, high signal intensity on T2-weighted images, and homogeneous enhancement on Gd-DTPA images. Neurilemmomas showed low signal intensity on T1-weighted images, high signal intensity on T2-weighted images, and variable enhancement on GdDTPA images. Nine neurilemmomas showed heterogeneous enhancement, and 3 cases showed rim enhancement. These variable enhancements corresponded to focal hemorrhage, focal necrosis, myxoid change, and cystic change on pathologic macroscopic findings (Fig. 1). 
Table 1. Histologic diagnosis and MR images

\begin{tabular}{|c|c|c|c|c|c|c|}
\hline \multirow{2}{*}{ Age } & \multirow{2}{*}{ Sex } & \multirow{2}{*}{ Level } & \multirow{2}{*}{$\mathrm{Bx}$} & \multicolumn{3}{|c|}{ MRI } \\
\hline & & & & $\mathrm{T} 1$ & $\mathrm{~T} 2$ & Gd-DTPA \\
\hline 33 & M & L3 & Neurilemoma & Low & High (heterogenous) & $\mathrm{O}$ \\
\hline 37 & M & $\mathrm{C} 1-2$ & Neurilemoma & Low & High & $\mathrm{O}$ (heterogenous) \\
\hline 41 & $\mathrm{~F}$ & L5 & Neurilemoma & Low & High & $\mathrm{O}$ (heterogenous) \\
\hline 36 & M & $\mathrm{S} 1$ & Neurilemoma & Low & High & $\mathrm{O}$ \\
\hline 67 & M & L2, L3-4 & Neurilemoma & Low & High & $\mathrm{O}$ \\
\hline 64 & $\mathrm{~F}$ & L5 & Neurilemoma & Low & High & $\mathrm{O}$ (heterogenous) \\
\hline 43 & M & L5-S1 & Neurilemoma & Low & High & $\mathrm{O}$ \\
\hline 37 & M & L2 & Neurilemoma & Low & High & $\mathrm{O}$ (heterogenous) \\
\hline 56 & $\mathrm{~F}$ & L3-4 & Neurilemoma & Low & High & $\mathrm{O}$ (heterogenous) \\
\hline 68 & $\mathrm{~F}$ & L1-2 & Neurilemoma & Low & High & $\mathrm{O}$ (rim) \\
\hline 34 & $\mathrm{~F}$ & Т9-10 & Neurilemoma & Low & High & $\mathrm{O}$ \\
\hline 33 & M & T6-7 & Neurilemoma & Low & High (heterogenous) & $\mathrm{O}$ \\
\hline 41 & M & $\mathrm{T} 12$ & Neurilemoma & Low & High & $\mathrm{O}$ (heterogenous) \\
\hline 69 & M & T9 & Neurilemoma & Low & High & $\mathrm{O}$ \\
\hline 29 & $\mathrm{~F}$ & T8-9 & Neurilemoma & Low & High & $\mathrm{O}$ (heterogenous) \\
\hline 37 & M & T12-L1 & Neurilemoma & Low & High & $\mathrm{O}$ (heterogenous) \\
\hline 45 & $\mathrm{~F}$ & L2-3 & Neurilemoma & Low & High & $\mathrm{O}$ (rim) \\
\hline 31 & $\mathrm{~F}$ & T12-L2 & Neurilemoma & Low & High & $\mathrm{O}$ (rim) \\
\hline 55 & $\mathrm{~F}$ & L4 & Neurilemoma & Low & High & $\mathrm{O}$ \\
\hline 69 & $\mathrm{~F}$ & $\mathrm{~T} 4-5$ & Meningioma & Iso & Low & $\mathrm{O}$ \\
\hline 62 & $\mathrm{~F}$ & S1-S3 & Meningioma & Iso & Iso & $\mathrm{O}$ \\
\hline 77 & $\mathrm{~F}$ & $\mathrm{~T} 4$ & Meningioma & Iso & Iso & $\mathrm{O}$ \\
\hline 55 & M & T5 & Meningioma & Iso & Iso & $\mathrm{O}$ \\
\hline 79 & $\mathrm{~F}$ & $\mathrm{~T} 10$ & Meningioma & Iso & Iso & $\mathrm{O}$ \\
\hline 33 & $\mathrm{~F}$ & $\mathrm{C} 3$ & Neurofibroma & Iso & High & $\mathrm{O}$ \\
\hline 8 & M & T10-11 & Neurofibroma & Intermediate & High & $\mathrm{O}$ \\
\hline 31 & $\mathrm{~F}$ & $\mathrm{~T} 7$ & Neurofibroma & Intermediate & High & $\mathrm{O}$ \\
\hline 39 & M & L4-5 & Neurofibroma & Iso & High & $\mathrm{O}$ \\
\hline 58 & $\mathrm{~F}$ & L2 & Hemangioma, carvenous & Slightly increase & High & $\mathrm{O}$ \\
\hline 50 & F & $\mathrm{T} 4-5$ & Hemangioma, carvenous & Slightly increase & High & $\mathrm{O}$ \\
\hline 59 & $\mathrm{~F}$ & L4 & Hemangioma, carvenous & Low & High & $\mathrm{O}$ \\
\hline & & & & Iso to slightly & Iso to slightly & \\
\hline 23 & M & L4-S2 & GCT of tendon sheath & Increase & Increase & $\mathrm{O}$ (heterogenous) \\
\hline 58 & $\mathrm{~F}$ & L5-S1 & Ganglioneuroma & Low & High & $\mathrm{O}$ \\
\hline 55 & M & $\mathrm{T} 4-5$ & Neuroblastoma & Low & Low & $\mathrm{O}$ \\
\hline 59 & M & $\mathrm{T} 4-7$ & Metastatic ca. (the prostate) & Intermediate & Intermediate & $\mathrm{O}$ \\
\hline 31 & M & C3-6 & Lymphoma & Low & High & $\mathrm{O}$ \\
\hline 24 & $\mathrm{~F}$ & L2-3 & AVM & Low & High & \\
\hline 28 & M & $\mathrm{S} 2$ & Arachnoid cyst & Low & High & \\
\hline 65 & $\mathrm{~F}$ & S1 & Arachnoid cyst & Low & High & \\
\hline
\end{tabular}

Gd-DTPA: gadolinium diethylene triamine acid, AVM: arteriovenous malformation, GCT: giant-cell tumor.

\section{Meningiomas}

Meningiomas comprise approximately $25 \%$ of primary intraspinal tumors and are second only to nerve sheath tumors in frequency. Spinal cord meningiomas are believed to originate from meningothelial cells near the distal root ganglia. Most of meningiomas are intradural extramedullary, and only 5\% are extradural. Five percent are intradural extramedullary and extradural in a dumbbell distribution. ${ }^{6}$

These tumors appear as rounded, sharply marginated masses that are isointense to the spinal cord on T1-weighted images and iso- or hypointense on T2-weighted images ${ }^{4}$. These tumors usually enhance intensely and homogeneously. The presence of tumor calcification may help to distin- 

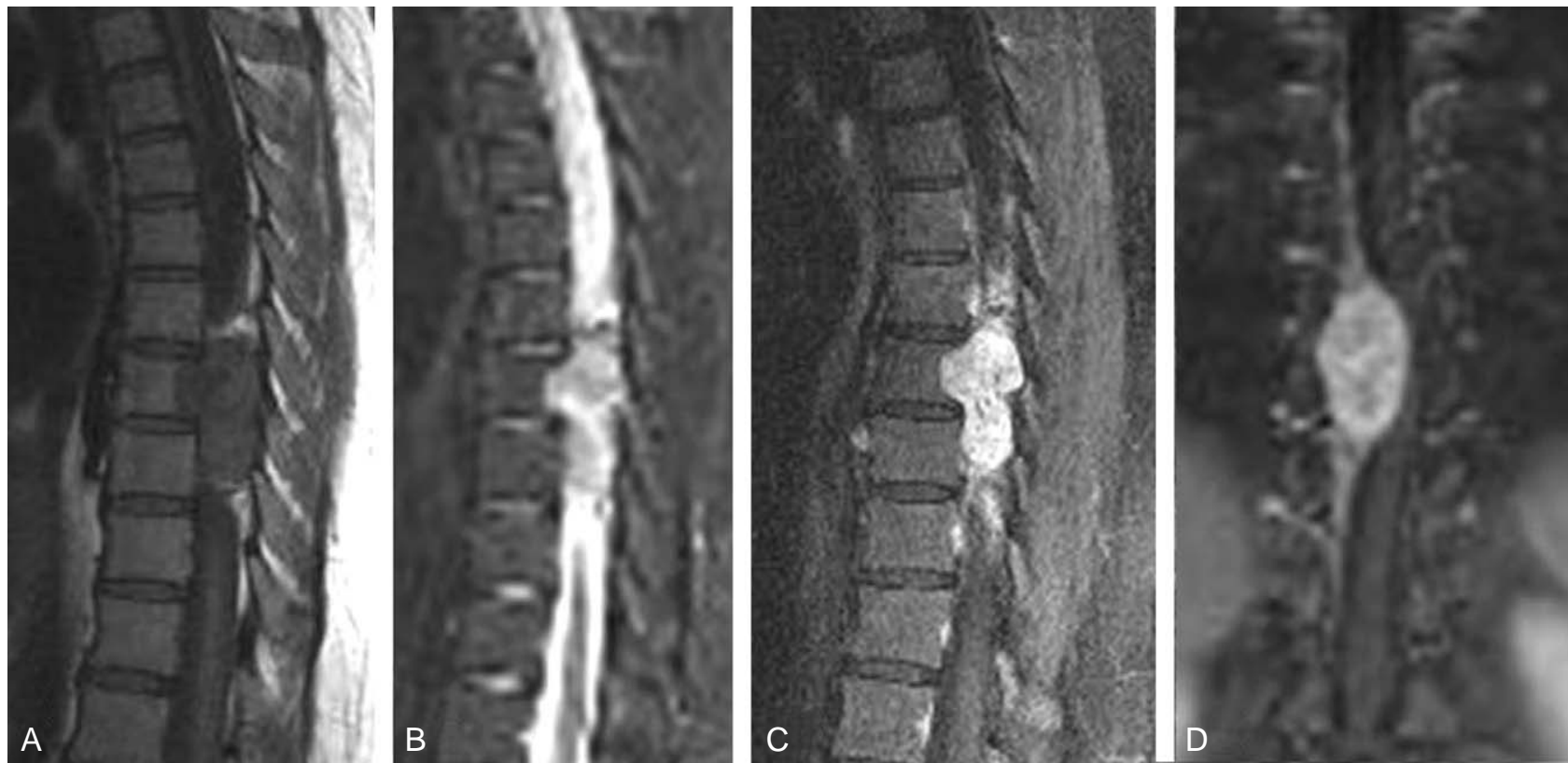

Fig. 1. A 30-year-old female patient who had undergone an operation for neurilemmoma demonstrated low signal intensity on T1weighted imaging (A), high signal intensity on T2-weighted imaging (B), and significant enhancement on Gd-DTPA imaging (C). Coronal MR imaging showed lateral displacement of the spinal cord (D). Gd-DTPA: gadolinium diethylene triamine acid.
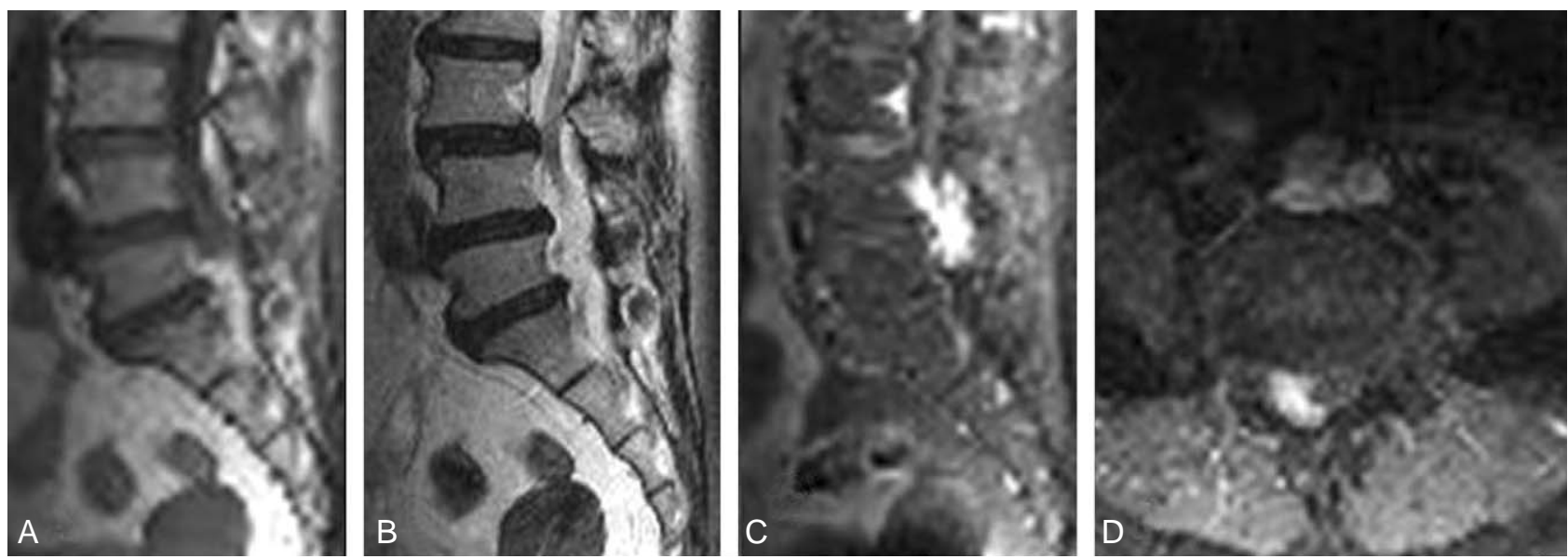

Fig. 2. A 61-year-old female patient who had undergone an operation for cavernous hemangioma demonstrated slightly increased signal intensity on T1-weighted imaging (A), high signal intensity on T2-weighted imaging (B), and significant enhancement on GdDTPA imaging (C). Axial MR imaging showed lateral displacement of the cauda equina (D). Gd-DTPA: gadolinium diethylene triamine acid.

guish it from nerve sheath tumors. Differentiation may also be determined by evaluating for neurofibromas that are heterogeneous on T2-weighted images and enhance more intensely than meningiomas do. In our cases, MR demonstrated isointense signal on T1-weighted images in all cases and low ( 1 case) or isointense (4 case) signal intensity on T2-weighted images. All cases demonstrated homogeneous enhancement on Gd-DTPA images and a solid portion on pathologic macroscopic findings.

\section{Hemangiomas}

Hemangiomas may arise from the blood vessels of the nerve roots in the cauda equina, the inner surface of the dura, or the pial surface of the spinal cord ${ }^{7}$. Capillary and cavernous hemangiomas usually behave as space-occupying masses, producing chronic progressive myelopathy or radiculopathy leading to motor and sensory deficits frequently associated with pain. There is a significant risk for bleeding, which may result in sudden neurologic deteriora- 
tion with associated hematomyelia or subarachnoid hemorrhage. Mastronardi et al. ${ }^{8}$ described a cavernous hemangioma of inhomogeneous hyperintensity on both T1- and T2-weighted images. The mixed high- and low- signal intensity components of cavernous hemangiomas presumably indicate the presence of mixed subacute and chronic hemorrhage 9 . In our cases, MR images demonstrated slightly increased and low signal intensity on T1-weighted images, high signal intensity on T2-weighted images, and homogeneous enhancement on Gd-DTPA images. The MRbased preoperative diagnosis was a neurogenic tumor, but the postoperative diagnosis was a cavernous hemangioma based on intraoperative and pathologic findings (Fig. 2).

\section{Lymphomas}

Lymphoma is not limited to a single spinal compartment. Spinal cord involvement usually reflects metastatic disease, and primary CNS lymphoma is uncommon in the spinal cord. Lymphoma demonstrates solid enhancement with adjacent high T2 signal intensity, consistent with edema. Cord enlargement is not as severe as with other intramedullary neoplasms. In our case, the pathologic diagnosis was primary dural lymphoma. MR images demonstrated low signal intensity on T1-weighted images, high signal intensity on T2-weighted images, and significant enhancement on GdDTPA images, with lateral displacement of the spinal cord. There were no signal changes detected in the spinal cord. The preoperative MR-based diagnosis was a neurogenic tumor, such as neurofibroma. Primary dural lymphoma is a rare subtype of primary central nervous system (CNS) lymphoma that arises from the dura mater and differs biologically from other CNS lymphomas. Primary dural lymphoma is usually a low-grade marginal zone lymphoma, whereas other types are usually high-grade, diffuse, large B-cell lymphomas. Primary dural lymphoma presents with diffuse enhancement and can be confused with a meningioma. They respond well to local surgical and radiation therapy ${ }^{10}$. The authors performed laminoplastic tumor excision.

\section{Ganglioneuromas}

Ganglioneuromas are rare benign tumors. Those developing within the spinal canal constitute less than 10 percent of all ganglioneuromas. Most cases involve the paraspinal region and intraspinal extension, leading to a dumbbellshaped tumor ${ }^{11}$. Other tumors occur within the spinal cord.
Rarely, intradural extramedullary ganglioneuromas have been reported. They occasionally grow to a large size, but total excision is possible under microscopic surgery. MR images characteristically show low signal intensity on T1weighted images and heterogeneous high signal intensity on T2-weighted images ${ }^{12}$. Though ganglioneuromas are typically avascular, hypertrophic vessels mimicking arteriovenous malformations have been described in spinal ganglioneuromas. In our case, a mass lesion was located in the L5 level spinal canal and in the left neural foramen along the nerve root. MR examination demonstrated low signal intensity on T1-weighted images, high signal intensity on T2-weighted images, and significant enhancement on Gd-DTPA images.

\section{Arachnoid cysts}

Arachnoid cysts occur as single or multiple cysts and are distributed in the spine as follows: $80 \%$ in the thoracic, $15 \%$ in the cervical, and 5\% in the lumbar spine. They affect men and women equally and are most commonly seen in patients between the ages of 30 and 50 years $^{13}$. Arachnoid cysts are usually located posterior to the thecal sac, and only rarely anteriorly ${ }^{14}$. MR imaging is the radiological test of choice, even though its interpretation may prove to be difficult in some instances, and the exact definition of the cyst's borders may remain unclear. Myelography and CT are not able to delineate the cyst in greater detail than MR images $^{15}$. In our cases, MR images demonstrated low signal intensity on T1-weighted images and high signal intensity on T2-weighted images, with sacral bone erosion at the S1 canal. Gd-DTPA enhancement was not performed.

\section{Giant cell tumors of tendon sheaths}

Giant cell tumors of tendon sheaths are lesions of uncertain histogenesis that occur in association with the synovial membrane of tendon sheaths, bursae, and joints. They frequently occur in the flexor tendons of the hands. Giant cell tumors of tendon sheaths in the spine are very rare. Spine involvement occurs predominantly in the lumbar or cervical spine $^{16}$. In our cases, MR images showed a mass that had isointense to slightly increased signal intensity relative to muscle on T1- and T2-weighted images, with inhomogeneous enhancement. Minoru et al reported that MR images commonly define a mass that is isointense relative to muscle on T1-weighted images and has variable intensity on T2-weighted images due to the presence of hemosiderin, 

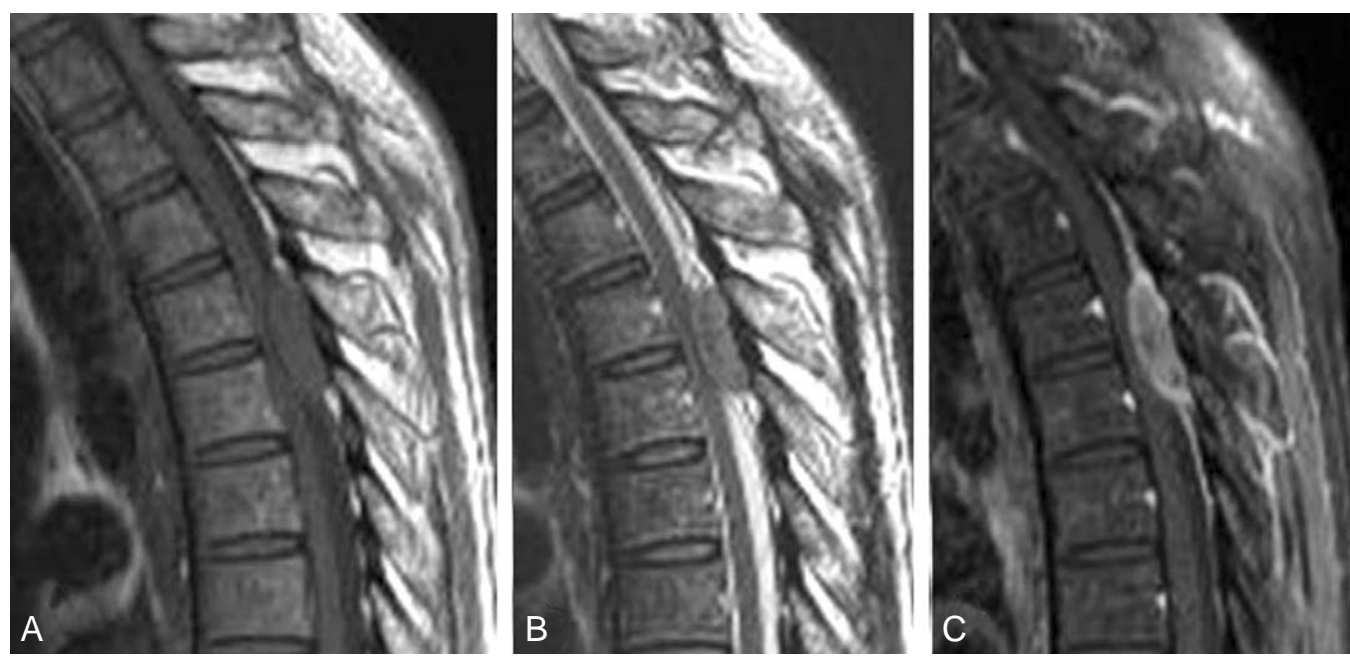

Fig. 3. A 55-year-old male patient who had undergone an operation for neuroblastoma demonstrated low signal intensity on T1weighted imaging (A), intermediate signal intensity on T2-weighted imaging (B), and inhomogeneous enhancement with high peripheral signal intensity on Gd-DTPA imaging (C). Gd-DTPA: gadolinium diethylene triamine acid.

cystic fluid, and hemorrhage ${ }^{17}$. Although these characteristics are by no means diagnostic for giant cell tumors of the tendon sheath, the amount of impingement on the spinal cord is best defined by MR images.

\section{Neuroblastomas}

Neuroblastomas invading the intervertebral foramen and the spinal canal typically exhibit a dumbbell appearance. The intraspinal component usually remains confined extradurally. MR imaging can detect the intraspinal extension of the mass. In our cases, neuroblastomas located in the epidural space of the spinal cord resulted in myelopathy. MR examination demonstrated low signal intensity on T1weighted imaging, intermediate signal intensity on T2weighted imaging, and inhomogeneous enhancement with peripheral high signal intensity on Gd-DTPA imaging (Fig. 3). Low signal intensity on T1-weighted images and intermediate intensity on T2-weighted images were due to high cellularity and high nuclear-to-cytoplasmic ratio. Necrotic cystic changes, hemorrhage, and calcification can cause heterogeneous signal behavior. Contrast enhancement is usually marked ${ }^{18}$. MR images also detect compression and displacement of the spinal cord. Intramedullary hyperintense signal on T2-weighted images reflects cord edema in the setting of compression-related myelopathy.

\section{Metastatic tumors}

Neoplasms that frequently metastasize to the bone struc- ture of the spine include myeloma, breast cancer, lung cancer, prostate cancer, and lymphoma. Osteoblastic metastases occur frequently in the setting of prostate or breast cancer. The thoracic spine is more commonly involved than the lumbar or cervical spine. MR is the best imaging modality for detecting and characterizing metastatic disease, but bone scintigraphy is more useful in detecting multiple lesions and distant metastases. MR imaging is useful for diagnosis, staging, evaluation of soft tissue complications, and treatment planning. Metastatic lesions show low signal intensity on T1-weighted images and high signal intensity on T2-weighted images. Sclerotic metastases may show low signal intensity on T2-weighted images ${ }^{2}$. Contrast enhancement is required to diagnose metastatic lesions if epidural disease is suspected. In our case, the metastatic lesion was derived from prostate cancer, and it had an osteoblastic appearance on plain radiography. MR images demonstrated diffuse heterogeneous osteoblastic metastases involving the entire thoracolumbar spine and sacrum, with epidural mass formation at T4-T7 and spinal cord compression. For relief of spinal cord compression, total laminoplastic tumor excision was performed on the epidural metastatic tumor.

\section{Conclusions}

MR images are the preoperative modality of choice in the evaluation of tumors of the spinal cord. Gd-DTPA images demonstrate variable enhancement. Awareness of the variegated presentation of these spinal cord tumors is important 
in forming a differential diagnosis and planning surgical resection.

\section{REFERENCES}

1. Brotchi J, Dewitte O, Levivier M, et al: A survey of 65 tumors within the spinal cord: surgical results and the importance of preoperative magnetic resonance imaging. Neurosurgery 1991; 29: 651-656.

2. Bloomer CW, Ackerman A, Bhatia RG, et al: Imaging for spine tumors and new applications. Top Magn Reson Imaging 2006; 17: 69-87.

3. Levy WJ, Latchaw J, Hahn JF, Sawhny B, Bay J, Dohn DF: Spinal neurofibromas: a report of 66 cases and a comparison with meningiomas. Neurosurgery 1986; 18: 331334.

4. Parizel PM, Baleriaux D, Rodesch G, et al: Gd-DTPAenhanced MR imaging of spinal tumors. AJR Am J Roentgenol 1989; 152: 1087-1096.

5. Friedman DP, Tartaglino LM, Flanders AE: Intradural schwannomas of the spine: MR findings with emphasis on contrast-enhancement characteristics. AJR Am J Roentgenol 1992; 158: 1347-1350.

6. Dickman CA, Fehlings MG, Gokaslan ZL: Spinal cord and spinal column tumors: principles and practice. New York, NY, Theme: 145-176, 2006.

7. Choi BY, Chang KH, Choe G, et al: Spinal intradural extramedullary capillary hemangioma: MR imaging findings. AJNR Am J Neuroradiol 2001; 22: 799-802.

8. Mastronardi L, Ferrante L, Scarpinati M, Gagliardi FM, Celli P, Fortuna A: Intradural extramedullary cavernous angioma: case report. Neurosurgery 1991; 29: 924-
926.

9. Fontaine S, Melanson D, Cosgrove R, Bertrand G: Cavernous hemangiomas of the spinal cord: MR imaging. Radiology 1988; 166: 839-841.

10. Iwamoto FM, Abrey LE: Primary dural lymphoma: a review. Neurosurg Focus 2006; 21: E5.

11. Oro JJ, Geise AW: Dumbbell ganglioneuroma of the lumbar spine associated with a herniated intervertebral disc: case report. Neurosurgery 1983; 13: 711-714.

12. Pang BC, Tchoyoson Lim CC, Tan KK: Giant spinal ganglioneuroma. J Clin Neurosci 2005; 12: 967-972.

13. Osenbach RK, Godersky JC, Traynelis VC, Schelper RD: Intradural extramedullary cysts of the spinal canal: clinical presentation, radiographic diagnosis, and surgical management. Neurosurgery 1992; 30: 35-42.

14. Spiegelmann R, Rapparport ZH, Sahar A: Spinal arachnoid cyst with unusual presentation. Case report. J Neurosurg 1984; 60: 613-616.

15. Wenger M, Zobor N, Markwalder R, Vogt E, Markwalder TM: Intradural extramedullary arachnoid cyst of the thoracic spine associated with cord compression. J Clin Neurosci 2007; 14: 693-696.

16. Dingle SR, Flynn JC, Flynn JC Jr, Stewart G: Giant-cell tumor of the tendon sheath involving the cervical spine. A case report. J Bone Joint Surg Am 2002; 84: 1664-1667.

17. Doita M, Miyamoto H, Nishisa K, Nabeshima Y, Yoshiya S, Kurosaka M: Giant-cell tumor of the tendon sheath involving the thoracic spine. J Spinal Disord Tech 2005; 18: 445-448.

18. De Bernardi B, Balwierz W, Bejent J, et al: Epidural compression in neuroblastoma: Diagnostic and therapeutic aspects. Cancer Lett 2005; 228: 283-299. 